\title{
Myocardial sympathetic denervation in Chagas' cardiomyopathy: A predictor of deterioration of left ventricular systolic function
}

\author{
Pieter van der Bijl, MD, PhD, ${ }^{a}$ and Jeroen J. Bax, MD, PhD ${ }^{\mathrm{a}, \mathrm{b}}$ \\ a Department of Cardiology, Heart Lung Center, Leiden University Medical Center, Leiden, The \\ Netherlands \\ b Heart Center, University of Turku and Turku University Hospital, Turku, Finland
}

Received Oct 18, 2021; accepted Oct 19, 2021

doi: $10.1007 / \mathrm{s} 12350-021-02854-w$

\section{See related article, pp. 3166-3176}

Chagas' disease is an anthropozoonosis caused by Trypanosoma cruzi, which is carried by triatomites. It is endemic only in the Americas, and although rare in the United States of America (USA), 300,000 infected individuals are estimated to live in the USA. The disease course comprises three phases: the acute, indeterminate and determinate phase. Cardiomyopathy rarely occurs in the acute phase, but is seen in up to $30 \%$ of individuals with chronic disease. The factors that trigger transition from the indeterminate phase to overt cardiomyopathy are largely unknown, although immunosuppression has been identified as a precipitant. Progression from the indeterminate to the determinate phase is characterized by myocardial inflammation, which eventually culminates in fibrosis. Once cardiomyopathy is clinically manifest, the prognosis is dismal: arrhythmias, heart failure, thromboembolism and sudden cardiac death occur in the overt phase of Chagas' cardiomyopathy. ${ }^{1}$ Treatment options for established Chagas' cardiomyopathy are limited, and essentially comprise standard anti-failure therapy. In the Evaluation of the Use of Antiparasital Drug in the Treatment of Chronic Chagas' Disease (BENEFIT) trial, benznidazole, a trypanocidal drug, did not reduce the primary combined endpoint of

Reprint requests: Pieter van der Bijl, MD, PhD, Department of Cardiology, Heart Lung Center, Leiden University Medical Center, Albinusdreef 2, 2300 RC Leiden, The Netherlands; pieter.vanderbijl@gmail.com

J Nucl Cardiol 2022;29:3177-8.

$1071-3581 / \$ 34.00$

Copyright ( 2021 American Society of Nuclear Cardiology. death, resuscitated cardiac arrest, sustained ventricular tachycardia, insertion of a pacemaker or implantable cardioverter-defibrillator, cardiac transplantation, new-onset heart failure, stroke or other thromboembolic events. ${ }^{2}$ Cardiac transplantation has demonstrated efficacy, ${ }^{3}$ but its application will always remain limited by donor availability. Taking into account the limited armamentarium available to clinicians to treat established disease, early markers of development or progression of Chagas' cardiomyopathy are required before strategies to prevent the evolution of this disease can be tested prospectively.

In the current issue of the Journal, Gadioli et al investigated the role of sympathetic innervation imaging in the prediction of development of left ventricular (LV) systolic dysfunction. The diagnosis of chronic Chagas' disease was confirmed with two different IgG assays, as recommended by the World Health Association. Eighteen patients with cardiac Chagas' disease were followed up for a mean of $5.5 \pm 1$ years from diagnosis. Baseline and follow-up scintigraphy were performed with ${ }^{99} \mathrm{mTc}$-sestamibi and ${ }^{123}$ I-metaiodobenzylguanidine (MIBG). LV ejection fraction (LVEF) at baseline and follow-up was quantified with transthoracic echocardiography, demonstrating a mean decrease in LVEF from $56 \pm 11 \%$ to $49 \pm 12 \%(P=.01)$. During the same time interval, the mean summed innervation score increased from $15 \pm 10$ to $20 \pm 9(P<.01)$. Viable but denervated LV regions at baseline were associated with a higher likelihood of developing regional wall motion abnormalities at follow-up in a regression analysis (OR 4.2; $P=.0002$ ).

The predictors of LVEF deterioration in Chagas' cardiomyopathy have not been extensively investigated. Myocardial perfusion abnormalities, demonstrated with scintigraphy, have been shown to precede LV systolic dysfunction. ${ }^{4}$ Sympathetic denervation, visualized with 
${ }^{123}$ I-MIBG, was found in areas of perfusion defects in patients with preserved LVEF and no regional wall motion abnormalities in a study of 37 individuals with varying severity of cardiomyopathy, ${ }^{5}$ thereby suggesting that denervation precedes LV systolic dysfunction. Cardiac sympathetic denervation has also been linked to ventricular tachycardias in Chagas' cardiomyopathy, which are known to be influenced by autonomic nervous system dysregulation. ${ }^{6}$

Early identification of patients with chronic Chagas' disease who are at risk of developing cardiomyopathy may allow the institution of therapy that will prevent the establishment of severe cardiac involvement or at least attenuate its progression. In view of the poor prognosis and limited treatment options of established cardiomyopathy, prevention is the single most auspicious approach that will allow a curtailment of the morbidity and mortality inherent to Chagas' cardiomyopathy. Early abnormalities have been detected on cardiac magnetic resonance (CMR) imaging, i.e., before the onset of LV dysfunction: early gadolinium enhancement, high signal on T2-weighted imaging ${ }^{7}$ and an exercise-induced decrease in the phosphocreatine-toadenosine triphosphate ratio decrease have been reported. ${ }^{8}$ These abnormalities however, have not been shown to predict disease progression. Speckle tracking echocardiography might prove to be more cost-effective than CMR as a screening tool for the development of cardiomyopathy in Chagas' disease. Scintigraphy is another potential imaging biomarker, and appears promising from the data presented in the current study. Although the deterioration of LV systolic function has not yet been linked to worse clinical outcome, such a link is certainly conceivable. Larger, multicenter studies will be required to investigate the role of imaging biomarkers in the early identification of Chagas' cardiomyopathy, before the development of overt disease. Ideally, this would include comparison between different imaging modalities, e.g., scintigraphy, echocardiography and CMR. Finally, prospective trials will be required to demonstrate which prophylactic approaches, guided by the identification of early disease with multimodality imaging, are effective in preventing or attenuating the progression of Chagas' cardiomyopathy.

\section{Disclosures}

The Department of Cardiology of Leiden University Medical Center received Research Grants from Abbott Vascular, Bayer, Biotronik, Bioventrix, Boston Scientific, Edwards Lifesciences, GE Healthcare and Medtronic. Jeroen Bax received speaker fees from Abbott Vascular and Edwards Lifesciences. Pieter van der Bijl has nothing to disclose.

\section{References}

1. Freitas HF, Chizzola PR, Paes AT, Lima AC, Mansur AJ. Risk stratification in a Brazilian hospital-based cohort of 1220 outpatients with heart failure: Role of Chagas' heart disease. Int J Cardiol 2005;102:239-47.

2. Morillo CA, Marin-Neto JA, Avezum A, Sosa-Estani S, Rassi A Jr, Rosas F, et al. Randomized trial of benznidazole for chronic Chagas' cardiomyopathy. N Engl J Med 2015;373:1295-306.

3. Bocchi EA, Fiorelli A. The paradox of survival results after heart transplantation for cardiomyopathy caused by Trypanosoma cruzi. First Guidelines Group for Heart Transplantation of the Brazilian Society of Cardiology. Ann Thorac Surg 2001;71:1833-8.

4. Hiss FC, Lascala TF, Maciel BC, Marin-Neto JA, Simoes MV. Changes in myocardial perfusion correlate with deterioration of left ventricular systolic function in chronic Chagas' cardiomyopathy. JACC Cardiovasc Imaging 2009;2:164-72.

5. Simoes MV, Pintya AO, Bromberg-Marin G, Sarabanda AV, Antloga CM, Pazin-Filho A, et al. Relation of regional sympathetic denervation and myocardial perfusion disturbance to wall motion impairment in Chagas' cardiomyopathy. Am J Cardiol 2000;86:975-81.

6. Miranda CH, Figueiredo AB, Maciel BC, Marin-Neto JA, Simoes MV. Sustained ventricular tachycardia is associated with regional myocardial sympathetic denervation assessed with ${ }^{123} \mathrm{I}-$ metaiodobenzylguanidine in chronic Chagas cardiomyopathy. J Nucl Med 2011;52:504-10.

7. Torreao JA, Ianni BM, Mady C, Naia E, Rassi CH, Nomura C, et al. Myocardial tissue characterization in Chagas' heart disease by cardiovascular magnetic resonance. J Cardiovasc Magn Reson 2015;17:97.

8. Betim Paes Leme AM, Salemi VM, Weiss RG, Parga JR, Ianni BM, Mady C, et al. Exercise-induced decrease in myocardial highenergy phosphate metabolites in patients with Chagas heart disease. J Card Fail 2013;19:454-60.

Publisher's Note Springer Nature remains neutral with regard to jurisdictional claims in published maps and institutional affiliations. 